\title{
The influence of substrate morphology on thickness uniformity and unintentional doping of epitaxial graphene on $\mathrm{SiC}$
}

Jens Eriksson, Ruth Pearce, Tihomir Iakimov, Chariya Virojanadara, Daniela Gogova, Mike Andersson, Mikael Syväjärvi, Anita Lloyd Spetz and Rositza Yakimova

\section{Linköping University Post Print}

N.B.: When citing this work, cite the original article.

Original Publication:

Jens Eriksson, Ruth Pearce, Tihomir Iakimov, Chariya Virojanadara, Daniela Gogova, Mike Andersson, Mikael Syväjärvi, Anita Lloyd Spetz and Rositza Yakimova, The influence of substrate morphology on thickness uniformity and unintentional doping of epitaxial graphene on SiC, 2012, Applied Physics Letters, (100), 24, 241607.

http://dx.doi.org/10.1063/1.4729556

Copyright: American Institute of Physics (AIP) http://www.aip.org/

Postprint available at: Linköping University Electronic Press http://urn.kb.se/resolve?urn=urn:nbn:se:liu:diva-78669 


\title{
The influence of substrate morphology on thickness uniformity and unintentional doping of epitaxial graphene on SiC
}

\author{
Jens Eriksson, ${ }^{1, a)}$ Ruth Pearce, ${ }^{1}$ Tihomir lakimov, ${ }^{1}$ Chariya Virojanadara, ${ }^{1}$ \\ Daniela Gogova, ${ }^{2}$ Mike Andersson, ${ }^{1}$ Mikael Syväjärvi, ${ }^{1}$ Anita Lloyd Spetz, ${ }^{1}$ \\ and Rositza Yakimova ${ }^{1}$ \\ ${ }^{1}$ Department of Physics, Chemistry and Biology, Linköping University, SE-58183 Linköping, Sweden \\ ${ }^{2}$ Leibniz Institute of Crystal Growth, 12489 Berlin, Germany
}

(Received 3 February 2012; accepted 31 May 2012; published online 15 June 2012)

\begin{abstract}
A pivotal issue for the fabrication of electronic devices on epitaxial graphene on $\mathrm{SiC}$ is controlling the number of layers and reducing localized thickness inhomogeneities. Of equal importance is to understand what governs the unintentional doping of the graphene from the substrate. The influence of substrate surface topography on these two issues was studied by work function measurements and local surface potential mapping. The carrier concentration and the uniformity of epitaxial graphene samples grown under identical conditions and on substrates of nominally identical orientation were both found to depend strongly on the terrace width of the SiC substrate after growth. (C 2012 American Institute of Physics. [http://dx.doi.org/10.1063/1.4729556]
\end{abstract}

Owing to favorable lattice matching, the preparation of graphene by thermal decomposition of $\mathrm{SiC}$ has emerged as a technology-friendly route for the growth of wafer-size epitaxial graphene (EG) layers for electronic device applications. ${ }^{1-3}$ Growth on insulating $\mathrm{SiC}$ substrates means that transfer to another insulator is not required. However, the electronic properties of EG are limited by thickness inhomogeneities and discontinuities in the grown films. ${ }^{4}$ Moreover, electronic interaction between the graphene and the $\mathrm{SiC}$ gives rise to an unintentional electron doping from the substrate, ${ }^{5}$ causing a shift of the Fermi level $\left(\mathrm{E}_{\mathrm{F}}\right)$ of up to $\approx 0.4 \mathrm{eV}$ above the Dirac point. ${ }^{6} \mathrm{~A}$ way of limiting this selfdoping by irradiation of Ar ions was recently reported by Jee et al., ${ }^{7}$ whereby this shift could be reduced to $0.2 \mathrm{eV}$. Still, achieving exact control over the self-doping remains an important hurdle to overcome for the fabrication of electronic devices on EG/SiC. Reports of carrier concentration in graphene on SiC vary depending on the growth conditions, ${ }^{8}$ but values deduced from Hall measurements show that in our material it is normally in the range of $10^{12} \mathrm{~cm}^{-2}$.

For large-scale device production homogeneous graphene thickness and accurate control over doping are important, especially for single- and bilayer graphene (1LG and 2LG), which have useful properties for device fabrication. Localized transport measurements based on scanning tunneling microscopy were recently employed to demonstrate the strong impact of atomic-scale substrate features on graphene performance. ${ }^{10}$ It was shown that scattering at surface steps and changes in layer thickness strongly reduce the macroscopic conductivity. A study by Robinson et al. ${ }^{11}$ found that the graphene uniformity and carrier concentration of graphene grown on $\mathrm{SiC}$ substrates are correlated with the $\mathrm{SiC}$ off-cut angles and the crystallographic orientation of the miscut. The same study further revealed large scattering in both the mobility and the carrier concentration for low miscut angles. We have observed a significant spread in the carrier

\footnotetext{
${ }^{\text {a) }}$ Author to whom correspondence should be addressed. Electronic mail: jenser@ifm.liu.se.
}

concentration of $\mathrm{EG}$ on $\mathrm{SiC}$ (0001) for samples grown under identical conditions on nominally on-axis SiC (0001) substrates. The aim of this study is therefore to further elucidate the influence of surface morphology on the electronic properties of epitaxial graphene, and in particular how it affects the thickness uniformity and the unintentional doping from the substrate. Due to the complex morphology of the $\mathrm{SiC}$ substrate, determination of local thickness variations using standard atomic force microscopy (AFM) morphology characterization is often infeasible. However, AFM coupled with electrostatic force measurements between the tip and the graphene sample (surface potential mapping) can be used to study nanoscale variations in the graphene homogeneity. ${ }^{12}$ In this study, scanning Kelvin probe microscopy (SKPM) is used to probe the local electronic state of graphene grown on $\mathrm{SiC}$ substrates with minute differences in the post-growth morphologies. These findings are complemented by macroscale results obtained from measurements in an ambient Kelvin probe.

The epitaxial graphene was prepared by sublimation of $\mathrm{SiC}$ and subsequent graphene formation on Si-terminated, semi-insulating (SI) $4 \mathrm{H}-\mathrm{SiC}$ or n-type $6 \mathrm{H}-\mathrm{SiC}(0001)$ onaxis substrates at $2000{ }^{\circ} \mathrm{C}$ in argon and at a pressure of 1 bar. $^{13,14}$ These conditions are conducive to fast surface kinetics due to the high temperature while also favoring a low rate of silicon loss from the surface, ${ }^{13,14}$ leading to larger areas of homogeneous graphene than high vacuum and ultrahigh vacuum (UHV) growth. ${ }^{13,15}$ A total of nine samples with slightly differing post-growth morphologies were investigated; their known parameters are listed in Table I.

The nanoscale morphological and electrical properties were determined at room temperature in lab ambient with a Veeco DI Dimension 3100 scanning probe microscope, equipped with the Nanoscope IV electronics. Surface potential maps were obtained by SKPM, which uses an interleave lift mode to record the surface potential. The measurements were performed using conductive, platinum coated Si tips (NT-MDT NSG01/Pt) with resonance frequencies between 98 and $154 \mathrm{kHz}$. To record the potential, the tip follows the 
TABLE I. Characteristics of the samples investigated in this study. The work function values are the average, large area, values measured in an ambient Kelvin probe (tip diameter of $0.5 \mathrm{~mm}$ ) and the value for 1LG has been calculated considering the potential difference between different number of graphene layers and the $1 \mathrm{LG} \%$.

\begin{tabular}{lcccccc}
\hline \hline Sample & $\begin{array}{c}\text { RMS } \\
(\mathrm{nm})\end{array}$ & $\begin{array}{c}\text { Terrace } \\
\text { width } \\
(\mathrm{nm})\end{array}$ & $\begin{array}{c}\text { Step } \\
\text { height }\end{array}$ & $1 \mathrm{LG} \%$ & Substrate & $\begin{array}{c}\Phi_{1 \mathrm{LG}} \\
(\mathrm{eV})\end{array}$ \\
\hline 1 & 1.31 & $849 \pm 20$ & $2.5 \pm 0.7$ & 73.3 & $6 \mathrm{H}-\mathrm{SiC}$ & 4.792 \\
2 & 0.24 & $947 \pm 81$ & $0.5 \pm 0.3$ & 87.2 & $4 \mathrm{H}-\mathrm{SiC}$ & 4.797 \\
3 & 0.24 & $1229 \pm 123$ & $0.5 \pm 0.1$ & 100 & $4 \mathrm{H}-\mathrm{SiC}$ & 4.951 \\
4 & 0.40 & $1400 \pm 440$ & $0.5 \pm 0.2$ & 55.7 & $4 \mathrm{H}-\mathrm{SiC}$ & 4.804 \\
5 & 0.54 & $830 \pm 150$ & $0.7 \pm 0.4$ & 88 & $6 \mathrm{H}-\mathrm{SiC}$ & 4.790 \\
6 & 0.73 & $330 \pm 50$ & $1 \pm 0.5$ & 37 & $6 \mathrm{H}-\mathrm{SiC}$ & 4.641 \\
7 & 0.36 & $660 \pm 60$ & $0.5 \pm 0.1$ & 79 & $4 \mathrm{H}-\mathrm{SiC}$ & 4.733 \\
8 & 0.76 & $1530 \pm 160$ & $0.9 \pm 0.5$ & 59 & $6 \mathrm{H}-\mathrm{SiC}$ & 4.819 \\
9 & 0.57 & $1850 \pm 500$ & $0.6 \pm 0.4$ & 51 & $4 \mathrm{H}-\mathrm{SiC}$ & 4.764 \\
\hline \hline
\end{tabular}

stored surface topography at a constant lift height of $10-20 \mathrm{~nm}$ above the sample while an ac bias of $3000 \mathrm{mV}$ is applied to the tip at the resonance frequency of the tip cantilever $(\omega)$. The tip DC bias is adjusted to nullify the tip oscillation at $\omega$, which is caused by the contact potential difference between the tip and the sample surface, $V_{C P D}$. The technique thus measures the work function difference, $\Phi_{\mathrm{S}}=\Phi_{\text {Tip }}-\mathrm{eV}_{\mathrm{CPD}}$, where $\Phi_{\mathrm{S}}$ and $\Phi_{\text {Tip }}$ are work functions of the sample surface and tip, respectively. $\mathrm{V}_{\mathrm{CPD}}$ is used to generate the SKPM data. The resulting image is effectively a map of the variations in the graphene work function, $\Phi_{\mathrm{G}}$.

The stability of SKPM measurements relies on the stability of the work function of the probe tips, and is sensitive to the measurement environment, such as humidity. Hence, the average values of $\Phi_{\mathrm{G}}$ were instead determined in an Ambient Kelvin probe from KP Technology, which determines $\Phi_{\mathrm{G}}$ over a much larger area (determined by the tip radius of $0.5 \mathrm{~mm}$ ). The work function of the tip is calibrated against a gold standard with a known work function and the values calculated are more stable as the tip never comes in contact with the sample, thereby avoiding contamination and tip deformation. Variations in, e.g., ambient humidity will influence the graphene work function significantly more than that of the gold reference electrode. As such, we focus on only the relative shift between different samples determined during the same measurement run (i.e., in the same environmental conditions).

Fig. 1 shows typical maps of morphology (a) and SKPM surface potential (b) for one of the investigated graphene/SiC samples (sample 5), along with the potential distribution (c). Terrace edges are visible on all samples and, depending on the sample, major step bunches are spaced between $\approx 0.3 \mu \mathrm{m}$ and $1.85 \mu \mathrm{m}$. The average terrace heights vary between samples in multiples of $\approx 0.5 \mathrm{~nm}$, corresponding roughly to two $\mathrm{SiC}$ bilayers, ranging from $0.5 \mathrm{~nm}$ to $2.5 \mathrm{~nm}$. Morphologyrelated parameters such as terrace width, step height, and surface roughness (RMS) for all samples are summarized in Table I.

As is seen in Fig. 1(b), only two discrete potentials can be observed (this is true for all investigated samples), where

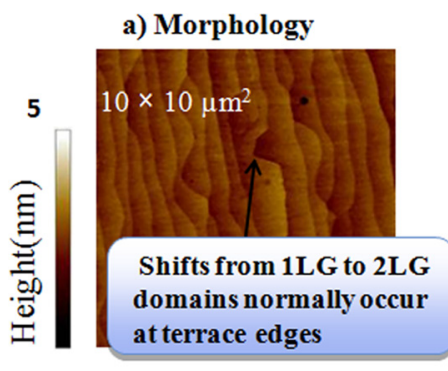

c) Potential distribution

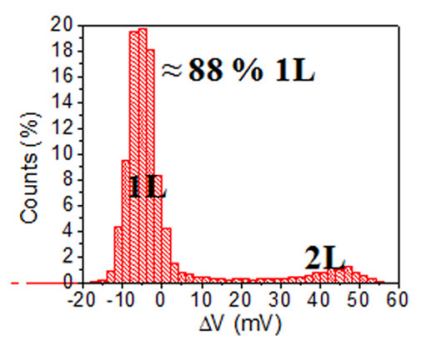

FIG. 1. AFM morphology (a) and SKPM surface potential map (b) of a graphene/SiC sample (sample 5); the SKPM potential distribution (averaged from three scans of $5 \times 5,10 \times 10$, and $25 \times 25 \mu \mathrm{m}^{2}$ areas), from which the monolayer coverage was evaluated, is shown in the histogram in (c); (d): LEEM image with a field of view of $50 \mu \mathrm{m}$, showing the distribution of $1 \mathrm{LG}$ and 2LG on sample 8. The superimposed SKPM potential maps measured on the same sample are in the same scale and show the same distribution of 1LG and 2LG, demonstrating that the SKPM technique correctly determines the graphene layer thickness.

the low potential corresponds to areas of $1 \mathrm{LG}$ and the higher potential areas are 2LG. $1 \mathrm{LG}$ to $2 \mathrm{LG}$ transitions are usually observed at step edges as can be seen for the sample in Fig. 1. The absence of thicker layers, or, of only the interfacial buffer layer in the samples was confirmed by either micro-Raman spectroscopy (spectra not shown here) performed with a $633 \mathrm{~nm}$ HeNe laser or low energy electron microscopy (LEEM). The accuracy of the layer thickness estimation using SKPM is demonstrated in Fig. 1(d) in which LEEM data are shown alongside SKPM potential maps measured on the same sample (sample 8). While the images do not show the exact same area, it is, however, clear that they show the same shape and distribution of $1 \mathrm{LG}$ and $2 \mathrm{LG}$. As such there is no doubt that SKPM correctly identifies the layer thickness.

The measured surface potential difference between areas of $1 \mathrm{LG}$ and $2 \mathrm{LG}$ is ascribed to different work functions for $1 \mathrm{LG}$ and $2 \mathrm{LG}$. Yu et al. ${ }^{16}$ showed that the work function of graphene increases gradually with increasing layer thickness, until saturation occurs at five layers of graphene due to interlayer screening. ${ }^{17}$ Hibino et al. ${ }^{18}$ later showed that this can be explained by a shift of the $\mathrm{C} 1 \mathrm{~s}$ core level towards lower binding energies as the number of layers increases. Consequently, the two observed potentials correspond to $1 \mathrm{LG}$ and 2LG, with the low potential corresponding to $1 \mathrm{LG}$. In this study, the potential difference between $1 \mathrm{LG}$ and $2 \mathrm{LG}$ is $25-50 \mathrm{mV}$, depending on the overall doping of the sample. This shift is smaller than that observed in UHV $(100-130 \mathrm{mV})^{16,18,19}$ but close to that observed under ambient conditions on exfoliated graphene $(\approx 66 \mathrm{mV}),{ }^{20}$ and in agreement with the values observed under ambient conditions for $\mathrm{EG}$ on $\mathrm{SiC}(0001)(\approx 25 \mathrm{mV}){ }^{21}$ 
Using histogram analysis, the 1LG surface coverage for each sample was approximated by calculating the area under a normal curve fitted to the 1LG peak in the potential distribution (Fig. 1(c)). For this analysis, the average distribution obtained from three scans of $5 \times 5,10 \times 10$, and $25 \times 25 \mu \mathrm{m}^{2}$ areas on each sample was used. A single peak potential distribution can be attributed either to a homogeneous $1 \mathrm{LG}$ or to interlayer charge screening due to multilayer graphene (MLG). In such cases the potential distribution is wider for the $1 \mathrm{LG}$, where the width of the distribution of $\approx 20-30 \mathrm{mV}$ can be attributed to an inhomogeneously doped background causing local changes to the carrier concentration in $1 \mathrm{LG}^{20}$ For MLG, the influence of charges in the substrate is attenuated due to the short interlayer screening length, ${ }^{17}$ which, coupled with the bulk-like behavior of MLG, results in a narrow and homogeneous distribution of $\approx 10 \mathrm{mV}$. However, no MLG was detected on the samples in this study. The $1 \mathrm{LG} \%$ for each sample is summarized in Table I.

From the data in Table I, we can identify two correlations, both concerning the average terrace width. Fig. 2 compares the $1 L G$ coverage and the terrace width. The homogeneity of the EG can be seen to strongly depend on the terrace width of the as-grown $\mathrm{EG} / \mathrm{SiC}$. As the distance between surface steps increases the area covered by monolayer graphene gradually increases until a terrace width of about $1200 \mathrm{~nm}$ is reached. This result is consistent with the growth of graphene beginning from the step edges; ${ }^{15}$ fewer steps result in fewer nucleation points and a reduction in bior multilayer growth. For terraces wider than $1200 \mathrm{~nm}$ the uniformity begins to decrease again, likely due to island growth in the absence of step edges. These results agree with previous findings of Robinson et al. ${ }^{11}$ However, that study looked into differences between graphene grown on $\mathrm{SiC}$ substrates of different off-cut angles and in different crystallographic orientations, whereas we focus on the morphology of the graphene-SiC surface after growth for substrates that have nominally identical orientation. Moreover, the growth conditions in the two studies are different, one study growing at $1625^{\circ} \mathrm{C}$ in $1 \mathrm{Torr}$ and this study growing at $2000^{\circ} \mathrm{C}$ in atmospheric pressure, whereby the growth kinetics, the

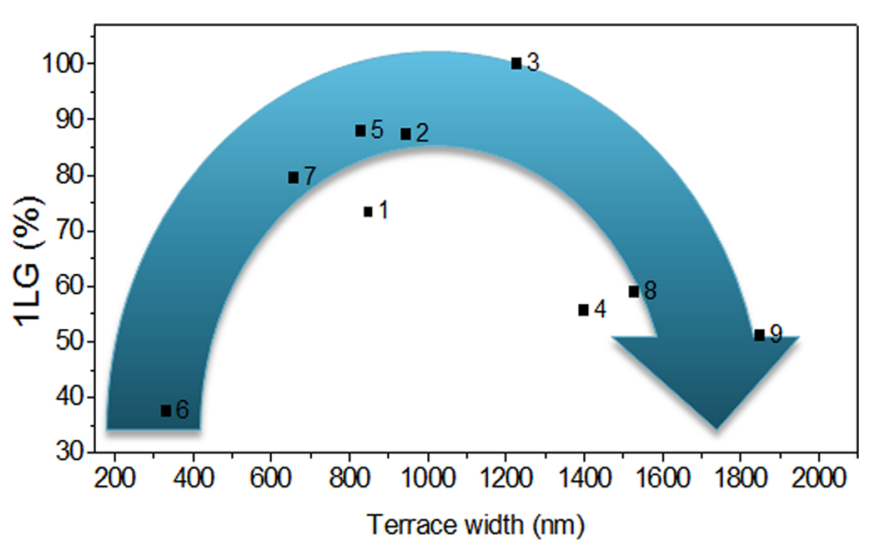

FIG. 2. Mono layer coverage vs. terrace width, showing that the homogeneity of the EG coverage strongly depends on the EG/SiC terrace width. As the terrace width increases towards $1200 \mathrm{~nm}$ the amount of 1LG gradually increases. For larger terraces, the 1LG\% drops off again. The numbers next to the data points correspond to the sample number as indicated in Table I. graphene-substrate interactions and the $\mathrm{SiC}$ restructuring are all different.

It may also be expected that the surface roughness would influence the number of layers. However, while the data in Table I suggest that a low RMS roughness does not reduce the likelihood of monolayer growth, there is no clear correlation as is observed in the case of the terrace width.

The data in Table I also enable evaluation of how the charge transfer from the $\mathrm{SiC}$ correlates with the graphene surface morphology. As the carrier concentration determines the position of $\mathrm{E}_{\mathrm{F}}$, which in turn determines $\Phi_{\mathrm{G}}$, relative shifts in the carrier concentration can be deduced. The $\Phi_{\mathrm{G}}$ values reported in Table I (4.64-4.95 eV) are higher than theoretical values for ideal graphene, and higher also than values obtained from measurements performed in UHV. This can be ascribed to p-type doping from the lab environment, since both $\mathrm{H}_{2} \mathrm{O}$ and $\mathrm{O}_{2}$ act as electron acceptors, ${ }^{22,23}$ lowering $\mathrm{E}_{\mathrm{F}}$ and thus, increasing $\Phi_{\mathrm{G}}$. Large changes $(\sim 200 \mathrm{meV})$ in the work function were observed from day to day due to chemical doping from adsorbents varying in the lab ambient. However, the work function differences between individual samples remain the same, suggesting that the different $\Phi_{\mathrm{G}}$ values measured on different samples are unrelated to adsorbents on the graphene surface, and should instead be explained by differing $\mathrm{E}_{\mathrm{F}}$ due to differing charge transfer from the buffer layer or the $\mathrm{SiC}$ substrate.

Fig. 3 shows the relative changes in $\Phi_{G}$ for $1 L G$ as a function of terrace width. All data were collected in one measurement run in order to avoid influences of changes in the lab ambient. $\Phi_{\mathrm{G}}$ for $1 \mathrm{LG}$ was calculated from the average, macro-scale work function considering the potential difference between different number of graphene layers and the 1LG\% measured by SKPM. Similar to the $1 \mathrm{LG}$ coverage, the work function gradually increases with increasing terrace width until about $1200 \mathrm{~nm}$ and then decreases again. This suggests the existence of an optimum terrace width near $1200 \mathrm{~nm}$ that will both minimize the unintentional doping and maximize the $1 \mathrm{LG}$ uniformity. Interestingly, the data in Table I suggest that the substrate polytype and doping (n-type $6 \mathrm{H}-\mathrm{SiC}$ or $\mathrm{SI} 4 \mathrm{H}-\mathrm{SiC}$ ) do not have any significant

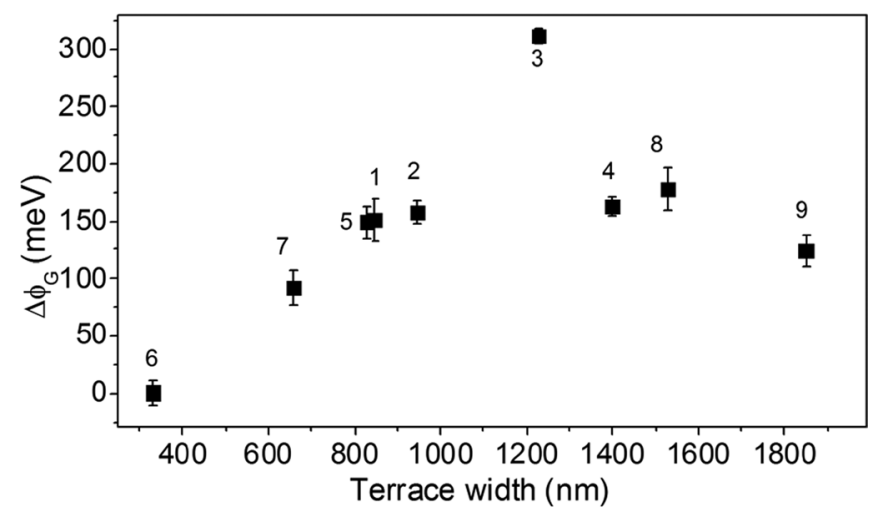

FIG. 3. Relative change in work function of $1 \mathrm{LG}$, measured in an ambient Kelvin probe, as a function of terrace width. All data were collected in one measurement run in order to avoid influences of changes in the lab ambient. The numbers next to the data points correspond to the sample number as indicated in Table I. Similar to the 1LG coverage, the work function gradually increases with increasing terrace width until about $1200 \mathrm{~nm}$ and then drops off again. 
influence on neither the uniformity nor the unintentional doping of the graphene.

Using the approximation that within $1 \mathrm{eV}$ of the Dirac point the carrier density, $\mathrm{N}$, relates to the linear density of states $\mathrm{D}_{0}=0.09$ (per $\mathrm{eV}^{2}$ unit cell), according to $\Delta \mathrm{N}(\mathrm{E})=\mathrm{D}_{0} \Delta \mathrm{E}_{\mathrm{F}}{ }^{2} / 2,{ }^{24}$ the observed spread in $\Phi_{\mathrm{G}} / \mathrm{E}_{\mathrm{F}}$ between the samples in this study corresponds to a maximum difference in carrier concentration of $8 \times 10^{12} \mathrm{~cm}^{-2}$, while the standard deviation in $\Phi_{\mathrm{G}} / \mathrm{E}_{\mathrm{F}}$ of $81.6 \mathrm{meV}$ translates to a carrier density standard deviation of $6 \times 10^{11} \mathrm{~cm}^{-2}$. However, this estimation does not consider that the density of states for EG on SiC may not be the same as for pristine graphene (where there is no strain and no interface states introduced by the EG/SiC buffer layer). Nevertheless, this result could explain the previously observed spread in the carrier concentration of EG for samples grown under identical conditions on nominally on-axis $\mathrm{SiC}$, as measured by Hall and sheet resistance techniques (not shown here).

As mentioned, the data in Table I suggest that the substrate doping does not significantly affect the unintentional doping of the as-grown graphene. This is in agreement with a previous study ${ }^{25}$ that showed that the carrier density of EG on $\mathrm{SiC}$ is not strongly influenced by the carrier concentration in the $\mathrm{SiC}$, and is instead thought more closely correlate with surface charges at the interface which could, in turn, be related to the substrate morphology. ${ }^{26}$ The differing $\Phi_{\mathrm{G}}$ values can thus likely be attributed to differing doping due to a surface step dependent charge transfer from the $\mathrm{SiC}$ substrate. Charges that are trapped between the 1LG and the $\mathrm{SiC}$ substrate are believed to aggregate at step edges, ${ }^{26}$ which is consistent with the observed trend (Fig. 3) of increasing $\Phi_{\mathrm{G}}$ (decreasing number of electrons) with increasing step distance within the range $300-1200 \mathrm{~nm}$. However, localized charge aggregation does not adequately fit with our experimental results. We do not observe significant local $\Phi_{\mathrm{G}}$ changes at the step edges, instead it appears that $\Phi_{\mathrm{G}}$ for $1 \mathrm{LG}$ is uniform over the whole sample despite differing significantly between samples. It should be noted, however, that minute and localized charge aggregations may still exist that cannot be measured due to limitations in the resolution of SKPM.

It has been demonstrated ${ }^{7}$ that contraction of the C-C bond length lowers the work function in graphene. Concomitantly, EG is compressively strained due to the difference in the thermal expansion coefficients of graphene and $\mathrm{SiC}^{27}$ Moreover, surface steps are associated with an increased uniform compressive strain in the graphene film. ${ }^{28}$ Consequently, the dependence of the carrier concentration on the step distance can likely be attributed to increased charge transfer from the $\mathrm{SiC}$ due to a strain-induced work function lowering in the graphene layer. Strain can be evaluated using micro-Raman spectroscopy; with a blue-shifted 2D peak indicating compressive strain. ${ }^{29}$ Quantitative estimation of the strain is difficult. Indeed, different dependencies of the 2D peak position on uniaxial strain are reported in literature, ranging from $21 \mathrm{~cm}^{-1} / \%$ (Ref. 30) to $66 \mathrm{~cm}^{-1} / \%{ }^{31}$ For our samples, the $2 \mathrm{D}$ peak position for $1 \mathrm{LG}$ differed by as much as $52 \mathrm{~cm}^{-1}\left(2692-2744 \mathrm{~cm}^{-1}\right)$ between samples, indicating significantly different strain levels. However, strain differences are not likely to account entirely for the measured $\Delta \Phi_{\mathrm{G}}$.
This study has allowed us to identify a target terrace width (around $\approx 1200 \mathrm{~nm}$ ) for as-grown epitaxial graphene on $\mathrm{SiC}$ that gives the optimal $1 \mathrm{LG} \%$ and that minimizes the unintentional doping. Before step-bunching occurs, the substrate step direction and terrace width are determined by the magnitude and direction of the surface misorientation. Consequently, a careful consideration of these parameters could yield command over terrace width and therefore the size and doping of the graphene domains. However, achieving control over the $\mathrm{SiC}$ restructuring during the high temperature growth is the prevalent obstacle on the path towards better controlled uniformity and electron doping in epitaxial graphene on $\mathrm{SiC}$. Consequently, future works are required with the aim of investigating how the surface of on-axis SiC substrates of differing step morphology and different polytype restructure during the sublimation growth, and how this affects the graphene formation.

In summary, we investigated the effect of minute differences in the surface morphologies of epitaxial graphene grown on nominally on-axis $\mathrm{SiC}$ substrates on the electronic properties of the graphene. It was found that the monolayer coverage depends strongly on the terrace width of the asgrown $\mathrm{EG} / \mathrm{SiC}$, where a more homogeneous coverage is favored by wider terraces in the range 300 to $\approx 1200 \mathrm{~nm}$. For wider terraces, the monolayer coverage begins to decrease. It was furthermore observed that the terrace width is a dominating factor in determining the unintentional doping of $1 \mathrm{LG}$ on SiC. Increasing the terrace width from $300 \mathrm{~nm}$ to $1200 \mathrm{~nm}$ resulted in an estimated reduction of n-type carriers by $8 \times 10^{12} \mathrm{~cm}^{-2}$. These results demonstrate that an important move towards the commercial growth of $\mathrm{EG}$ on $\mathrm{SiC}$ is to achieve more accurate control over the restructuring of the $\mathrm{SiC}$ surface during high temperature graphene growth.

We acknowledge support from the Swedish Government Strategic Research Area in Materials Science on Functional Materials at Linköping University (Faculty Grant SFO-MatLiU \# 2009-00971).

${ }^{1}$ C. Berger, Z. Song, T. Li, X. Li, A. Y. Ogbazghi, R. Feng, Z. Dai, A. N. Marchenkov, E. H. Conrad, P. N. First, and W. A. de Heer, J. Phys. Chem. B 108, 19912-19916 (2004).

${ }^{2}$ C. Berger, Z. Song, X. Li, X. Wu, N. Brown, C. Naud, D. Mayou, T. Li, J Hass, A. N. Marchenkov, E. H. Conrad, P. N. First, and W. A. de Heer, Science 312, 1191-1196 (2006).

${ }^{3}$ T. Ohta, A. Bostwick, Th. Seyller, K. Horn, and E. Rotenberg, Science 313, 951-954 (2006).

${ }^{4}$ T. Ohta, F. El Gabaly, A. Bostwick, J. L. McChesney, K. V. Emtsev, A. K. Schmid, Th. Seyller, K. Horn, and E. Rotenberg, New J. Phys. 10, 023034 (2008).

${ }^{5}$ S. Kopylov, A. Tzalenchuk, S. Kubatkin, and V. I. Fal'Ko, Appl. Phys. Lett. 97, 112109 (2010)

${ }^{6}$ S. Y. Zhou, G.-H. Gweon, A. V. Fedorov, P. N. First, W. A. de Heer, D.-H. Lee, F. Guinea, A. H. C. Neto, and A. Lanzara, Nature Mater. 6, 770 (2007).

${ }^{7}$ H.-G. Jee, K.-H. Jin, J.-H. Han, H.-N. Hwang, S.-H. Jhi, Y.-D. Kim, and C.-C. Hwang, Phys. Rev. B 84, 075457 (2011).

${ }^{8}$ J. L. Tedesco, B. L. VanMil, R. L. Myers-Ward, J. M. McCrate, S. A. Kitt, P. M. Campbell, G. G. Jernigan, J. C. Culbertson, C. R. Eddy, and D. K. Gaskill, Appl. Phys. Lett. 95, 122102 (2009).

${ }^{9}$ A. Tzalenchuk, S. Lara-Avila, A. Kalaboukhov, S. Paolillo, M. Syväjärvi, R. Yakimova, O. Kazakova, T. J. B. M. Janssen, V. Fal'ko, and S. Kubatkin, Nat. Nanotechnol. 5, 186-189 (2010).

${ }^{10}$ S.-H. Ji, J. B. Hannon, R. M. Tromp, V. Perebeinos, J. Tersoff, and F. M. Ross, Nature Mater. 11, 114-119 (2012). 
${ }^{11}$ J. A. Robinson, K. A. Trumbull, M. LaBella, R. Cavalero, M. J. Hollander, M. Zhu, M. T. Wetherington, M. Fanton, and D. W. Snyder, Appl. Phys. Lett. 98, 222109 (2011).

${ }^{12}$ T. Burnett, R. Yakimova, and O. Kazakova, Nano Lett.11, 2324-2328 (2011).

${ }^{13}$ C. Virojanadara, M. Syväjarvi, R. Yakimova, and L. I. Johansson, Phys. Rev. B 78, 245403 (2008).

${ }^{14}$ R. Yakimova, C. Virojanadara, D. Gogova, M. Syväjärvi, D. Siche, K. Larsson, and L. I. Johansson, Mater. Sci. Forum 565-568, 645-648 (2010).

${ }^{15}$ K. V. Emtsev, A. Bostwick, K. Horn, J. Jobst, G. L. Kellogg, L. Ley, J. L. McChesney, T. Ohta, S. A. Reshanov, J. Röhrl, E. Rotenberg, A. K. Schmid, D. Waldmann, H. B. Weber, and Th. Seyller, Nature Mater. 8, 203 (2009).

${ }^{16}$ Y. J. Yu, Y. Zhao, S. Ryu, L. E. Brus, K. S. Kim, and P. Kim, Nano Lett. 9, 3430-3434 (2009).

${ }^{17}$ Guinea, F. Phys. Rev. B 75, 235433 (2007).

${ }^{18}$ H. Hibino, H. Kageshima, M. Kotsugi, F. Maeda, F. Z. Guo, and Y. Watanabe, Phys. Rev. B 79, 125437 (2009).

${ }^{19}$ T. Filleter, K. V. Emtsev, Th. Seyller, and R. Bennewitz, Appl. Phys. Lett. 93, 133117 (2008).

${ }^{20}$ D. Ziegler, P. Gava, J. Güttinger, F. Molitor, L. Wirtz, M. Lazzeri, A. M. Saitta, A. Stemmer, F. Mauri, and C. Stampfer, Phys. Rev. B 83, 235434 (2011).
${ }^{21}$ A. E. Curtin, M. S. Fuhrer, J. L. Tedesco, R. L. Myers-Ward, C. R. Eddy, Jr., and D. K. Gaskill, Appl. Phys. Lett. 98, 243111 (2011).

${ }^{22}$ S. J. Sque, R. Jones, and P. R. Briddon, Phys. Status Solidi A 204, 3078 (2007).

${ }^{23}$ F. Schedin, K. Geim, S. K. Morozov, E. W. Hill, M. I. Blake, P. Katsnelson, and K. S. Novoselov, Nature Mater. 6, 652 (2007).

${ }^{24}$ G. Giovannetti, P. A. Khomyakov, G. Brocks, V. M. Karpan, J. van den Brink, and P. J. Kelly, Phys. Rev. Lett. 101, 026803 (2008).

${ }^{25}$ S. Y. Zhou, G.-H. Gweon, A. V. Fedorov, P. N. First, W. A. De Heer, D.-H. Lee, and F. Guinea, Nature Mater. 6, 770-775 (2007).

${ }^{26}$ M. K. Yakes, D. Gunlycke, J. L. Tedesco, P. M. Campbell, R. L. MyersWard, C. R. Eddy, Jr., D. K. Gaskill, P. E. Sheehan, and A. R. Laracuente, Nano Lett. 10, 1559-1562 (2010).

${ }^{27}$ N. Ferralis, R. Maboudian, and C. Carraro, Phys. Rev. Lett. 101, 156801 (2008).

${ }^{28}$ N. Ferralis, J. Kawasaki, R. Maboudian, and C. Carraro, Appl. Phys. Lett. 93, 191916 (2008).

${ }^{29}$ J. A. Robinson, C. P. Puls, N. E. Staley, J. P. Stitt, M. A. Fanton, K. V. Emtsev, Th. Seyller, and Y. Liu, Nano Lett. 9, 964-968 (2009).

${ }^{30}$ M. Huang, H. Yan, C. Chen, D. Song, T. F. Heinz, and J. Hone, Proc. Natl. Acad. Sci. U.S.A. 106, 7304-7308 (2009).

${ }^{31}$ G. Tsoukleri, J. Parthenios, K. Papagelis, R. Jalil, A. C. Ferrari, A. K. Geim, K. S. Novoselov, and C. Galiotis, Small 5, 2397-2402 (2009). 